This is a post-peer-review, pre-copyedit version of an article published in Annali di Matematica Pura ed Applicata. Series IV. The final authenticated version is available online at: http://dx.doi.org/10.1007/s10231-017-0671-2.

\title{
ON NEARLY SASAKIAN AND NEARLY COSYMPLECTIC MANIFOLDS
}

\author{
ANTONIO DE NICOLA, GIULIA DILEO, AND IVAN YUDIN
}

\begin{abstract}
We prove that every nearly Sasakian manifold of dimension greater than five is Sasakian. This provides a new criterion for an almost contact metric manifold to be Sasakian. Moreover, we classify nearly cosymplectic manifolds of dimension greater than five.
\end{abstract}

\section{INTRODUCTION}

One of the most successful attempts to relax the definition of a Kähler manifold is provided by the notion of a nearly Kähler manifold. Namely, nearly Kähler manifolds are defined as almost Hermitian manifolds $(M, J, g)$ such that the covariant derivative of the almost complex structure with respect to the Levi-Civita connection is skew-symmetric, that is

$$
\left(\nabla_{X} J\right) X=0,
$$

for every vector field $X$ on $M$. A remarkable classification of nearly Kähler manifolds was obtained by Nagy in [14]. This result reveals how 6-dimensional nearly Kähler manifolds play a central role, appearing as one of the possible factors in the de Rham decomposition of a complete simply connected strict nearly Kähler manifold.

Notice that in the defining condition of a nearly Kähler manifold, only the symmetric part of $\nabla J$ vanishes, in contrast to the Kähler case where $\nabla J=0$. Nearly Sasakian and nearly cosymplectic manifolds were defined in the same spirit starting from Sasakian and coKähler (sometimes also called cosymplectic) manifolds, respectively.

A smooth manifold $M$ endowed with an almost contact metric structure $(\phi, \xi, \eta, g)$ is said to be nearly Sasakian if

$$
\left(\nabla_{X} \phi\right) X=g(X, X) \xi-\eta(X) X
$$

for every vector field $X$ on $M$. Similarly, the condition for $M$ to be nearly cosymplectic is given by

$$
\left(\nabla_{X} \phi\right) X=0
$$

for every vector field $X$ on $M$.

The notion of a nearly Sasakian manifold was introduced by Blair and his collaborators in 4], while nearly cosymplectic manifolds were studied by Blair and Showers in [1, 3]. In the subsequent literature on the topic, quite important were the papers of Olszak [15, 16] for nearly Sasakian manifolds and those of Endo [9, 10]

2000 Mathematics Subject Classification. Primary 53C25, 53D35.

This work was partially supported by CMUC - UID/MAT/00324/2013, funded by the Portuguese Government through FCT/MEC and co-funded by the European Regional Development Fund through the Partnership Agreement PT2020 (A.D.N. and I.Y.), by MICINN (Spain) grants MTM2012-34478 (A.D.N.), and by the exploratory research project in the frame of Programa Investigador FCT IF/00016/2013 (I.Y.). G. Dileo thanks the Centre for Mathematics of the University of Coimbra for the hospitality received. 
on nearly cosymplectic manifolds. Later on, these two classes have played a role in the Chinea-Gonzalez's classification of almost contact metric manifolds ([8]). They also appeared in the study of harmonic almost contact structures (cf. [11, [17]). In [13, Loubeau and Vergara-Diaz proved that a nearly cosymplectic structure, once identified with a section of a twistor bundle, always defines a harmonic map.

Recently, a systematic study of nearly Sasakian and nearly cosymplectic manifolds was carried forward in [7. In that paper, the authors proved that any nearly Sasakian manifold is a contact manifold. In the 5-dimensional case, they showed that any nearly Sasakian manifold admits a nearly hypo $S U(2)$-structure that can be deformed to give a Sasaki-Einstein structure. Moreover, they proved that any nearly Sasakian manifold of dimension 5 has an associated nearly cosymplectic structure, thereby showing the close relation between these two notions. For 5dimensional nearly cosymplectic manifolds, they proved that any such manifold is Einstein with positive scalar curvature. It is also worth remarking that (1parameter families of) examples of both nearly Sasakian and nearly cosymplectic structures are provided by every 5-dimensional manifold endowed with a SasakiEinstein $S U(2)$-structure.

While Sasakian manifolds are characterized by the equality

$$
\left(\nabla_{X} \phi\right) Y=g(X, Y) \xi-\eta(Y) X
$$

the defining condition (1) of a nearly Sasakian manifold gives a constraint only on the symmetric part of $\nabla \phi$. In this paper we show that, surprisingly, in dimension higher than five, condition (1) is enough for the manifold to be Sasakian.

Concerning nearly cosymplectic manifolds, we prove that a nearly cosymplectic non-coKähler manifold $M$ of dimension $2 n+1>5$ is locally isometric to one of the following Riemannian products:

$$
\mathbb{R} \times N^{2 n}, \quad M^{5} \times N^{2 n-4},
$$

where $N^{2 n}$ is a nearly Kähler non-Kähler manifold, $N^{2 n-4}$ is a nearly Kähler manifold, and $M^{5}$ is a nearly cosymplectic non-coKähler manifold. If one makes the further assumption that the manifold is complete and simply connected, then the isometry becomes global.

\section{DEFinitions AND KNOWN RESUltS}

An almost contact metric manifold is a differentiable manifold $M$ of odd dimension $2 n+1$, endowed with a structure $(\phi, \xi, \eta, g)$, given by a tensor field $\phi$ of type $(1,1)$, a vector field $\xi$, a 1-form $\eta$ and a Riemannian metric $g$ satisfying

$$
\phi^{2}=-I+\eta \otimes \xi, \quad \eta(\xi)=1, \quad g(\phi X, \phi Y)=g(X, Y)-\eta(X) \eta(Y)
$$

for all vector fields $X, Y$ on $M$ (see [2, 5] for further details). From the definition it follows that $\phi \xi=0$ and $\eta \circ \phi=0$. Moreover, $\phi$ is skew-symmetric with respect to $g$, so that the bilinear form $\Phi:=g(-, \phi-)$ defines a 2 -form on $M$, called fundamental 2 -form. An almost contact metric manifold such that $d \eta=2 \Phi$ is called a contact metric manifold. In this case $\eta$ is a contact form, i.e. $\eta \wedge(d \eta)^{n} \neq 0$ everywhere on $M$.

A Sasakian manifold is defined as a contact metric manifold such that the tensor field $N_{\phi}:=[\phi, \phi]+d \eta \otimes \xi$ vanishes identically. It is well known that an almost contact metric manifold is Sasakian if and only if the Levi-Civita connection satisfies:

$$
\left(\nabla_{X} \phi\right) Y=g(X, Y) \xi-\eta(Y) X \text {. }
$$

A nearly Sasakian manifold is an almost contact metric manifold $(M, \phi, \xi, \eta, g)$ such that

$$
\left(\nabla_{X} \phi\right) Y+\left(\nabla_{Y} \phi\right) X=2 g(X, Y) \xi-\eta(X) Y-\eta(Y) X
$$


for all vector fields $X, Y$ on $M$, or, equivalently, (1) is satisfied.

We recall some basic facts about nearly Sasakian manifolds. We refer to 4, 15 , [16, 7] for the details.

In any nearly Sasakian manifold $(M, \phi, \xi, \eta, g)$, the characteristic vector field $\xi$ is Killing and the Levi-Civita connection satisfies $\nabla_{\xi} \xi=0$ and $\nabla_{\xi} \eta=0$. One can define a tensor field $h$ of type $(1,1)$ by putting

$$
\nabla_{X} \xi=-\phi X+h X
$$

The operator $h$ is skew-symmetric and anticommutes with $\phi$. It satisfies $h \xi=0$, $\eta \circ h=0$ and

$$
\nabla_{\xi} h=\nabla_{\xi} \phi=\phi h=\frac{1}{3} \mathcal{L}_{\xi} \phi
$$

where $\mathcal{L}_{\xi}$ denotes the Lie derivative with respect to $\xi$. The vanishing of $h$ provides a necessary and sufficient condition for a nearly Sasakian manifold to be Sasakian (16]). In [15] the following formulas are proved:

(6) $g\left(\left(\nabla_{X} \phi\right) Y, h Z\right)=\eta(Y) g\left(h^{2} X, \phi Z\right)-\eta(X) g\left(h^{2} Y, \phi Z\right)+\eta(Y) g(h X, Z)$,

$$
\begin{gathered}
\left(\nabla_{X} h^{2}\right) Y=\eta(Y)(\phi-h) h^{2} X+g\left((\phi-h) h^{2} X, Y\right) \xi \\
R(\xi, X) Y=\left(\nabla_{X} \phi\right) Y-\left(\nabla_{X} h\right) Y=g\left(X-h^{2} X, Y\right) \xi-\eta(Y)\left(X-h^{2} X\right),
\end{gathered}
$$

where $R$ is the Riemannian curvature of $g$.

A central role in the study of nearly Sasakian geometry is played by the symmetric operator $h^{2}$. We recall the fundamental result due to Olszak [15]:

Theorem 2.1. If a nearly Sasakian non-Sasakian manifold $(M, \phi, \xi, \eta, g)$ satisfies the condition

$$
h^{2}=\lambda(I-\eta \otimes \xi)
$$

for some real number $\lambda$, then $\operatorname{dim}(M)=5$.

In [16. Olszak also proved that any 5-dimensional nearly Sasakian non-Sasakian manifold is Einstein with scalar curvature $>20$. In 7 it is proved that the eigenvalues of $h^{2}$ are constant. Being $h$ skew-symmetric, the non-vanishing eigenvalues of $h^{2}$ are negative, so that the spectrum of $h^{2}$ is of type

$$
\operatorname{Spec}\left(h^{2}\right)=\left\{0,-\lambda_{1}^{2}, \ldots,-\lambda_{r}^{2}\right\},
$$

$\lambda_{i} \neq 0$ and $\lambda_{i} \neq \lambda_{j}$ for $i \neq j$. Further, if $X$ is an eigenvector of $h^{2}$ with eigenvalue $-\lambda_{i}^{2}$, then $X, \phi X, h X, h \phi X$ are orthogonal eigenvectors of $h^{2}$ with eigenvalue $-\lambda_{i}^{2}$. Hence the minimum dimension for a nearly Sasakian non-Sasakian manifold is 5 . In the following we denote by $[\xi]$ the 1-dimensional distribution generated by $\xi$, and by $\mathcal{D}(0)$ and $\mathcal{D}\left(-\lambda_{i}^{2}\right)$ the distributions of the eigenvectors 0 and $-\lambda_{i}^{2}$ respectively. We shall also denote by $\overline{\mathcal{D}}$ the distribution $[\xi] \oplus \mathcal{D}\left(-\lambda_{1}^{2}\right) \oplus \cdots \oplus \mathcal{D}\left(-\lambda_{r}^{2}\right)$, and by $\mathcal{D}_{0}$ the distribution orthogonal to $\overline{\mathcal{D}}$, so that $\mathcal{D}(0)=[\xi] \oplus \mathcal{D}_{0}$.

We will use the following results, proved in [7, concerning nearly Sasakian manifolds of dimension $\geq 5$.

Theorem 2.2. Let $M$ be a nearly Sasakian manifold with structure $(\phi, \xi, \eta, g)$ and let $\operatorname{Spec}\left(h^{2}\right)=\left\{0,-\lambda_{1}^{2}, \ldots,-\lambda_{r}^{2}\right\}$ be the spectrum of $h^{2}$. Then the distributions $\mathcal{D}(0)$ and $[\xi] \oplus \mathcal{D}\left(-\lambda_{i}^{2}\right)$ are integrable with totally geodesic leaves. In particular,

a) the eigenvalue 0 has multiplicity $2 p+1, p \geq 0$. If $p>0$, the leaves of $\mathcal{D}(0)$ are $(2 p+1)$-dimensional Sasakian manifolds;

b) each negative eigenvalue $-\lambda_{i}^{2}$ has multiplicity 4 and the leaves of the distribution $[\xi] \oplus \mathcal{D}\left(-\lambda_{i}^{2}\right)$ are 5-dimensional nearly Sasakian (non-Sasakian) manifolds. 
c) If $p>0$, the distribution $\overline{\mathcal{D}}=[\xi] \oplus \mathcal{D}\left(-\lambda_{1}^{2}\right) \oplus \cdots \oplus \mathcal{D}\left(-\lambda_{r}^{2}\right)$ is integrable with totally geodesic leaves.

Theorem 2.3. For a nearly Sasakian manifold $(M, \phi, \xi, \eta, g)$ of dimension $2 n+1 \geq$ 5 the 1-form $\eta$ is a contact form.

Before listing some known results on nearly cosymplectic manifolds, we recall that an almost contact metric manifold $(M, \phi, \xi, \eta, g)$ is said to be a coKähler manifold if $d \eta=0, d \Phi=0$ and $N_{\phi} \equiv 0$. Equivalently, one can require $\nabla \phi=0$. It is known that a coKähler manifold is locally the Riemannian product of the real line and a Kähler manifold, which is an integral submanifold of the distribution $\mathcal{D}=\operatorname{Ker}(\eta)$. Note that some authors call cosymplectic the class of manifold that we denominate coKähler (see [6] for details).

A nearly cosymplectic manifold is an almost contact metric manifold $(M, \phi, \xi, \eta, g)$ such that

$$
\left(\nabla_{X} \phi\right) Y+\left(\nabla_{Y} \phi\right) X=0
$$

for all vector fields $X, Y$. Clearly, this condition is equivalent to (2). It is known that in a nearly cosymplectic manifold the Reeb vector field $\xi$ is Killing and satisfies $\nabla_{\xi} \xi=0$ and $\nabla_{\xi} \eta=0$. The tensor field $h$ of type $(1,1)$ defined by

$$
\nabla_{X} \xi=h X
$$

is skew-symmetric and anticommutes with $\phi$. It satisfies $h \xi=0, \eta \circ h=0$ and

$$
\nabla_{\xi} \phi=\phi h=\frac{1}{3} \mathcal{L}_{\xi} \phi
$$

The following formulas hold $([9,10])$ :

$$
\begin{aligned}
g\left(\left(\nabla_{X} \phi\right) Y, h Z\right) & =\eta(Y) g\left(h^{2} X, \phi Z\right)-\eta(X) g\left(h^{2} Y, \phi Z\right), \\
\left(\nabla_{X} h\right) Y & =g\left(h^{2} X, Y\right) \xi-\eta(Y) h^{2} X, \\
\operatorname{tr}\left(h^{2}\right) & =\text { constant. }
\end{aligned}
$$

\section{Nearly Sasakian manifoldS}

We start by computing the covariant derivatives of the structure endomorphisms $\phi$ and $h$ on a nearly Sasakian manifold.

Proposition 3.1. Let $(M, \phi, \xi, \eta, g)$ be a nearly Sasakian manifold of dimension $2 n+1 \geq 5$. Then for all vector fields $X, Y$ on $M$ one has

$$
\begin{array}{cc}
(14) & \left(\nabla_{X} \phi\right) Y=\eta(X) \phi h Y-\eta(Y)(X+\phi h X)+g(X+\phi h X, Y) \xi, \\
(15) & \left(\nabla_{X} h\right) Y=\eta(X) \phi h Y-\eta(Y)\left(h^{2} X+\phi h X\right)+g\left(h^{2} X+\phi h X, Y\right) \xi, \\
(16) & \left(\nabla_{X} \phi h\right) Y=g\left(\phi h^{2} X-h X, Y\right) \xi+\eta(X)\left(\phi h^{2} Y-h Y\right)-\eta(Y)\left(\phi h^{2} X-h X\right) .
\end{array}
$$

Proof. From (6), for all vector fields $X, Y, Z$ we have

$$
g\left(\left(\nabla_{X} \phi\right) Y, h Z\right)=-\eta(Y) g(\phi h X, h Z)+\eta(X) g(\phi h Y, h Z)-\eta(Y) g(X, h Z),
$$

which is coherent with (14). On the other hand,

$$
\begin{aligned}
g\left(\left(\nabla_{X} \phi\right) Y, \xi\right) & =-g\left(Y,\left(\nabla_{X} \phi\right) \xi\right)=g\left(Y, \phi \nabla_{X} \xi\right)=g\left(Y,-\phi^{2} X+\phi h X\right) \\
& =g(X+\phi h X, Y)-\eta(X) \eta(Y) .
\end{aligned}
$$

Now, assume that $\operatorname{Spec}\left(h^{2}\right)=\left\{0,-\lambda_{1}^{2}, \ldots,-\lambda_{r}^{2}\right\}$ and consider the distribution $\overline{\mathcal{D}}=$ $[\xi] \oplus \mathcal{D}\left(-\lambda_{1}^{2}\right) \oplus \cdots \oplus \mathcal{D}\left(-\lambda_{r}^{2}\right)$. In order to complete the proof of [14), it remains to show that

$$
g\left(\left(\nabla_{X} \phi\right) Y, V\right)=-\eta(Y) g(X, V)
$$


for every $X, Y \in \mathfrak{X}(M)$ and $V \in \mathcal{D}_{0}$. Since the distribution $\overline{\mathcal{D}}$ is integrable with totally geodesic leaves, if $X, Y \in \overline{\mathcal{D}}$ then $\left(\nabla_{X} \phi\right) Y \in \overline{\mathcal{D}}$ and both sides in (17) vanish. Now consider $X \in \mathcal{D}_{0}$ and $Y \in \overline{\mathcal{D}}$. Then

$$
g\left(\left(\nabla_{X} \phi\right) Y, V\right)=-g\left(Y,\left(\nabla_{X} \phi\right) V\right)=-\eta(Y) g(X, V),
$$

where we applied the fact that the distribution $\mathcal{D}(0)=[\xi] \oplus \mathcal{D}_{0}$ is integrable with totally geodesic leaves, and the induced almost contact metric structure on each leaf is Sasakian, so that $\left(\nabla_{X} \phi\right) V=g(X, V) \xi-\eta(V) X$. On the other hand, if we take $X \in \overline{\mathcal{D}}$ and $Y \in \mathcal{D}_{0}$, then $g\left(\left(\nabla_{Y} \phi\right) X, V\right)=-\eta(X) g(Y, V)$, and applying (4), we have

$$
g\left(\left(\nabla_{X} \phi\right) Y, V\right)=-g\left(\left(\nabla_{Y} \phi\right) X+\eta(X) Y, V\right)=0 .
$$

Finally, taking $X, Y \in \mathcal{D}_{0},(17)$ is verified because of (3) and the fact that the vector fields $X, Y, V$ are orthogonal to $\xi$.

As regards (15), it follows from (8) and (14). Finally, a straightforward computation using (14) and (15) gives (16).

We will write $\epsilon_{d \eta}$ for the operator on $\Omega^{*}(M)$ defined by $\omega \mapsto d \eta \wedge \omega$.

Proposition 3.2. Let $(M, \eta)$ be a contact manifold of dimension $2 n+1$. Then, the operator

$$
\begin{aligned}
\epsilon_{d \eta}: \Omega^{2}(M) & \rightarrow \Omega^{4}(M) \\
\beta & \mapsto d \eta \wedge \beta
\end{aligned}
$$

is injective for $n \geq 3$.

Proof. Since $d \eta$ is a nondegenerate 2 -form on the distribution $\mathcal{D}=\operatorname{Ker}(\eta)$, the assumption $n \geq 3$ implies that the operators

$$
\epsilon_{d \eta}: \Omega^{1}(\mathcal{D}) \rightarrow \Omega^{3}(\mathcal{D}) \quad \alpha \mapsto d \eta \wedge \alpha
$$

and

$$
\epsilon_{d \eta}: \Omega^{2}(\mathcal{D}) \rightarrow \Omega^{4}(\mathcal{D}) \quad \beta \mapsto d \eta \wedge \beta .
$$

are injective. For every $k \geq 1$ we have

$$
\Omega^{k}(M)=\Omega^{k}(\mathcal{D}) \oplus \eta \wedge \Omega^{k-1}(\mathcal{D}) .
$$

Indeed, every $k$-form $\omega$ on $M$ can be decomposed as

$$
\omega=i_{\xi}(\eta \wedge \omega)+\eta \wedge i_{\xi} \omega .
$$

On the other hand, if a $k$-form $\omega$ belongs to the intersection of the two subspaces, that is $\omega \in \Omega^{k}(\mathcal{D})$ and $\omega=\eta \wedge \sigma$, with $\sigma \in \Omega^{k-1}(\mathcal{D})$, then

$$
\sigma=i_{\xi}(\eta \wedge \sigma)+\eta \wedge i_{\xi} \sigma=i_{\xi} \omega=0,
$$

and thus $\omega=0$. This shows that the sum in 20 is direct.

Now, let $\omega=\beta+\eta \wedge \alpha$, with $\beta \in \Omega^{2}(\mathcal{D})$ and $\alpha \in \Omega^{1}(\mathcal{D})$, be a 2 -form on $M$ such that $d \eta \wedge \omega=0$. Then, owing to (20) for $k=4$, we have $d \eta \wedge \beta=0$ and $d \eta \wedge \eta \wedge \alpha=0$, which also gives $d \eta \wedge \alpha=0$. Finally, we deduce from the injectivity of the operators in 180 and $(19)$ that both the forms $\beta$ and $\alpha$ vanish, and thus $\omega=0$.

Now we are able to prove our main result.

Theorem 3.3. Every nearly Sasakian manifold of dimension $2 n+1>5$ is Sasakian. 
Proof. Let $M$ be a nearly Sasakian manifold with structure $(\phi, \xi, \eta, g)$, of dimension $2 n+1$. We consider the 2 -forms $H$ and $\Phi_{k}, k=1,2$, defined by

$$
H(X, Y)=g(h X, Y), \quad \Phi_{k}(X, Y)=g\left(\phi h^{k} X, Y\right) .
$$

We shall prove that

$$
\begin{aligned}
d H & =3 \eta \wedge \Phi_{1}, \\
d \Phi_{1} & =3 \eta \wedge\left(\Phi_{2}-H\right) .
\end{aligned}
$$

From 15, we have that for all vector fields $X, Y, Z$,

$$
\begin{aligned}
g\left(\left(\nabla_{X} h\right) Y, Z\right)= & \eta(X) g(\phi h Y, Z)-\eta(Y) g\left(h^{2} X+\phi h X, Z\right)+\eta(Z) g\left(h^{2} X+\phi h X, Y\right) \\
= & \eta(X) g(\phi h Y, Z)+\eta(Y) g(\phi h Z, X)+\eta(Z) g(\phi h X, Y) \\
& -\eta(Y) g\left(h^{2} Z, X\right)+\eta(Z) g\left(h^{2} X, Y\right) .
\end{aligned}
$$

Therefore,

$$
\begin{aligned}
d H(X, Y, Z) & =g\left(\left(\nabla_{X} h\right) Y, Z\right)+g\left(\left(\nabla_{Y} h\right) Z, X\right)+g\left(\left(\nabla_{Z} h\right) X, Y\right) \\
& =3(\eta(X) g(\phi h Y, Z)+\eta(Y) g(\phi h Z, X)+\eta(Z) g(\phi h X, Y)) \\
& =3 \eta \wedge \Phi_{1}(X, Y, Z) .
\end{aligned}
$$

Analogously, from (16), we have

$$
\begin{aligned}
g\left(\left(\nabla_{X} \phi h\right) Y, Z\right)= & \eta(X) g\left(\phi h^{2} Y-h Y, Z\right)-\eta(Y) g\left(\phi h^{2} X-h X, Z\right) \\
& +\eta(Z) g\left(\phi h^{2} X-h X, Y\right) \\
= & \eta(X) g\left(\phi h^{2} Y, Z\right)+\eta(Y) g\left(\phi h^{2} Z, X\right)+\eta(Z) g\left(\phi h^{2} X, Y\right) \\
& -\eta(X) g(h Y, Z)-\eta(Y) g(h Z, X)-\eta(Z) g(h X, Y) .
\end{aligned}
$$

Hence,

$$
\begin{aligned}
d \Phi_{1}(X, Y, Z)= & g\left(\left(\nabla_{X} \phi h\right) Y, Z\right)+g\left(\left(\nabla_{Y} \phi h\right) Z, X\right)+g\left(\left(\nabla_{Z} \phi h\right) X, Y\right) \\
= & 3\left(\eta(X) g\left(\phi h^{2} Y, Z\right)+\eta(Y) g\left(\phi h^{2} Z, X\right)+\eta(Z) g\left(\phi h^{2} X, Y\right)\right) \\
& -3(\eta(X) g(h Y, Z)+\eta(Y) g(h Z, X)+\eta(Z) g(h X, Y)) \\
= & 3 \eta \wedge \Phi_{2}(X, Y, Z)-3 \eta \wedge H(X, Y, Z) .
\end{aligned}
$$

Now, from 21) and (22), we have

$$
0=d^{2} H=3 d \eta \wedge \Phi_{1}-3 \eta \wedge d \Phi_{1}=3 d \eta \wedge \Phi_{1} .
$$

If we assume that the dimension of $M$ is $2 n+1>5, \eta$ being a contact form, the fact that $d \eta \wedge \Phi_{1}=0$ implies $\Phi_{1}=0$, by Proposition 3.2. Therefore $h=0$, and the structure is Sasakian.

\section{NeARLy COSYMPLECTIC MANIFOLDS}

In this section we will classify nearly cosymplectic manifolds of dimension higher than five. In the following, given a nearly cosymplectic manifold $(M, \phi, \xi, \eta, g)$, we shall denote by $h$ the operator defined in 100 .

Proposition 4.1. Let $(M, \phi, \xi, \eta, g)$ be a nearly cosymplectic manifold. Then $h=0$ if and only if $M$ is locally isometric to the Riemannian product $\mathbb{R} \times N$, where $N$ is a nearly Kähler manifold.

Proof. For every vector fields $X, Y$ we have

$$
d \eta(X, Y)=g\left(\nabla_{X} \xi, Y\right)-g\left(\nabla_{Y} \xi, X\right)=2 g(h X, Y) .
$$

Therefore, if $h=0$ the distribution $\mathcal{D}=\operatorname{Ker}(\eta)$ is integrable. Denoting by $N$ an integral submanifold of $\mathcal{D}$, it is a totally geodesic hypersurface of $M$. Indeed, for every $X, Y \in \mathcal{D}$, we have $g\left(\nabla_{X} Y, \xi\right)=-g(Y, h X)=0$. Being also $\nabla_{\xi} \xi=0, M$ 
turns out to be locally isometric to the Riemannian product $\mathbb{R} \times N$. Further, the almost contact metric structure induces on $N$ an almost Hermitian structure which is nearly Kähler.

Conversely, if $M$ is locally isometric to the Riemannian product $\mathbb{R} \times N$, where $N$ is a nearly Kähler manifold, then $d \eta(X, Y)=0$ for all vector fields $X, Y$ orthogonal to $\xi$. By 23 and $h \xi=0$, we deduce that $h=0$.

As a consequence of the above proposition, a nearly cosymplectic manifold $(M, \phi, \xi, \eta, g)$ is coKähler if and only if $h=0$ and the leaves of the distribution $\mathcal{D}$ are Kähler manifolds. Recall that 4-dimensional nearly Kähler manifolds are Kähler (see [12, Theorem 5.1]), and this implies that if $M$ is a 5-dimensional nearly cosymplectic manifold with $h=0$, then it is a coKähler manifold.

We shall now study the spectrum of the symmetric operator $h^{2}$.

Proposition 4.2. The eigenvalues of the symmetric operator $h^{2}$ are constant.

Proof. From 12 it follows that

$$
\left(\nabla_{X} h^{2}\right) Y=g\left(X, h^{3} Y\right) \xi-\eta(Y) h^{3} X .
$$

Let us consider an eigenvalue $\mu$ of $h^{2}$ and a local unit vector field $Y$, orthogonal to $\xi$, such that $h^{2} Y=\mu Y$. Applying (24) for any vector field $X$, we have

$$
\begin{aligned}
0 & =g\left(\left(\nabla_{X} h^{2}\right) Y, Y\right) \\
& =g\left(\nabla_{X}\left(h^{2} Y\right), Y\right)-g\left(h^{2}\left(\nabla_{X} Y\right), Y\right) \\
& =X(\mu) g(Y, Y)+\mu g\left(\nabla_{X} Y, Y\right)-g\left(\nabla_{X} Y, h^{2} Y\right) \\
& =X(\mu) g(Y, Y)
\end{aligned}
$$

which implies that $X(\mu)=0$.

Since $h$ is skew-symmetric, the non-vanishing eigenvalues of $h^{2}$ are negative. Therefore, the spectrum of $h^{2}$ is of type

$$
\operatorname{Spec}\left(h^{2}\right)=\left\{0,-\lambda_{1}^{2}, \ldots,-\lambda_{r}^{2}\right\},
$$

where we can assume that each $\lambda_{i}$ is a positive real number and $\lambda_{i} \neq \lambda_{j}$ for $i \neq j$. Notice that if $X$ is an eigenvector of $h^{2}$ with eigenvalue $-\lambda_{i}^{2}$, then $X, \phi X, h X$, $h \phi X$ are orthogonal eigenvectors of $h^{2}$ with eigenvalue $-\lambda_{i}^{2}$. Since $h(\xi)=0$, we get the eigenvalue 0 has multiplicity $2 p+1$ for some integer $p \geq 0$.

We denote by $\mathcal{D}(0)$ the distribution of the eigenvectors with eigenvalue 0 , and by $\mathcal{D}_{0}$ the distribution of the eigenvectors in $\mathcal{D}(0)$ orthogonal to $\xi$, so that $\mathcal{D}(0)=$ $[\xi] \oplus \mathcal{D}_{0}$. Let $\mathcal{D}\left(-\lambda_{i}^{2}\right)$ be the distribution of the eigenvectors with eigenvalue $-\lambda_{i}^{2}$. We remark that the distributions $\mathcal{D}_{0}$ and $\mathcal{D}\left(-\lambda_{i}^{2}\right)$ are $\phi$-invariant and $h$-invariant.

Proposition 4.3. Let $(M, \phi, \xi, \eta, g)$ be a nearly cosymplectic manifold and let $\operatorname{Spec}\left(h^{2}\right)=\left\{0,-\lambda_{1}^{2}, \ldots,-\lambda_{r}^{2}\right\}$ be the spectrum of $h^{2}$. Then,

(a) for each $i=1, \ldots, r$, the distribution $[\xi] \oplus \mathcal{D}\left(-\lambda_{i}^{2}\right)$ is integrable with totally geodesic leaves.

Assuming that the eigenvalue 0 is not simple,

(b) the distribution $\mathcal{D}_{0}$ is integrable with totally geodesic leaves, and each leaf of $\mathcal{D}_{0}$ is endowed with a nearly Kähler structure;

(c) the distribution $[\xi] \oplus \mathcal{D}\left(-\lambda_{1}^{2}\right) \oplus \ldots \oplus \mathcal{D}\left(-\lambda_{r}^{2}\right)$ is integrable with totally geodesic leaves. 
Proof. Consider an eigenvector $X$ of $h^{2}$ with eigenvalue $-\lambda_{i}^{2}$. Then $\nabla_{X} \xi=h X \in$ $\mathcal{D}\left(-\lambda_{i}^{2}\right)$. On the other hand, (24) implies that $\nabla_{\xi} h^{2}=0$, and thus $\nabla_{\xi} X$ is also an eigenvector with eigenvalue $-\lambda_{i}^{2}$. Now, taking $X, Y \in \mathcal{D}\left(-\lambda_{i}^{2}\right)$ and applying (24), we get

$$
h^{2}\left(\nabla_{X} Y\right)=-\lambda_{i}^{2} \nabla_{X} Y-\left(\nabla_{X} h^{2}\right) Y=-\lambda_{i}^{2} \nabla_{X} Y+\lambda_{i}^{2} g(X, h Y) \xi .
$$

Therefore,

$$
h^{2}\left(\phi^{2} \nabla_{X} Y\right)=\phi^{2}\left(h^{2} \nabla_{X} Y\right)=-\lambda_{i}^{2} \phi^{2}\left(\nabla_{X} Y\right) .
$$

Thus $\phi^{2} \nabla_{X} Y \in \mathcal{D}\left(-\lambda_{i}^{2}\right)$. It follows that $\nabla_{X} Y=-\phi^{2} \nabla_{X} Y+\eta\left(\nabla_{X} Y\right) \xi$ belongs to the distribution $[\xi] \oplus \mathcal{D}\left(-\lambda_{i}^{2}\right)$. This proves $(a)$.

As regards (b), applying again (24), we have $\left(\nabla_{X} h^{2}\right) Y=0$ for every $X, Y \in \mathcal{D}_{0}$, so that $h^{2}\left(\nabla_{X} Y\right)=0$. Moreover,

$$
g\left(\nabla_{X} Y, \xi\right)=-g\left(Y, \nabla_{X} \xi\right)=-g(Y, h X)=0 .
$$

Hence, $\mathcal{D}_{0}$ is integrable with totally geodesic leaves. Since the leaves of $\mathcal{D}_{0}$ are $\phi$-invariant, the nearly cosymplectic structure induces a nearly Kähler structure on each integral submanifold of $\mathcal{D}_{0}$.

Finally, in order to prove $(c)$, owing to $(a)$, we only have to show that

$$
g\left(\nabla_{X} Y, Z\right)=0
$$

for every $X \in \mathcal{D}\left(-\lambda_{i}^{2}\right), Y \in \mathcal{D}\left(-\lambda_{j}^{2}\right), i \neq j$, and $Z \in \mathcal{D}_{0}$. In fact, from 24, we have

$$
\begin{aligned}
g\left(\nabla_{X} Y, Z\right) & =-\frac{1}{\lambda_{j}^{2}} g\left(\nabla_{X}\left(h^{2} Y\right), Z\right) \\
& =-\frac{1}{\lambda_{j}^{2}} g\left(\left(\nabla_{X} h^{2}\right) Y+h^{2}\left(\nabla_{X} Y\right), Z\right) \\
& =-\frac{1}{\lambda_{j}^{2}} \eta(Z) g\left(X, h^{3} Y\right)-\frac{1}{\lambda_{j}^{2}} g\left(\nabla_{X} Y, h^{2} Z\right)
\end{aligned}
$$

which vanishes since $\eta(Z)=0$ and $h^{2} Z=0$.

Theorem 4.4. Let $(M, \phi, \xi, \eta, g)$ be a nearly cosymplectic manifold such that 0 is a simple eigenvalue of $h^{2}$. Then $M$ is a 5-dimensional manifold.

Proof. First we show that

$$
\begin{aligned}
\left(\nabla_{X} \phi\right) Y & =g(\phi h X, Y) \xi+\eta(X) \phi h Y-\eta(Y) \phi h X, \\
\left(\nabla_{X} \phi h\right) Y & =g\left(\phi h^{2} X, Y\right) \xi+\eta(X) \phi h^{2} Y-\eta(Y) \phi h^{2} X
\end{aligned}
$$

for all vector fields $X$ and $Y$. Applying (10) we have

$$
g\left(\left(\nabla_{X} \phi\right) Y, \xi\right)=-g\left(Y,\left(\nabla_{X} \phi\right) \xi\right)=g\left(Y, \phi \nabla_{X} \xi\right)=g(Y, \phi h X) .
$$

Taking a vector field $U$ orthogonal to $\xi$, then $U=h Z$ for some vector field $Z$. Then, by applying (11) and recalling that $\phi$ anticommutes with $h$, we get

$$
\begin{aligned}
g\left(\left(\nabla_{X} \phi\right) Y, U\right) & =\eta(Y) g\left(h^{2} X, \phi Z\right)-\eta(X) g\left(h^{2} Y, \phi Z\right) \\
& =\eta(Y) g(h X, \phi h Z)-\eta(X) g(h Y, \phi h Z) \\
& =-\eta(Y) g(\phi h X, U)+\eta(X) g(\phi h Y, U)
\end{aligned}
$$

which completes the proof of 25$)$. From $(12)$ and $(25)$ we easily get 26 .

We consider now the 2 -forms $\Phi_{k}, k=0,1,2$, defined by

$$
\Phi_{k}(X, Y)=g\left(\phi h^{k} X, Y\right) .
$$

In particular, $\Phi_{0}=-\Phi$. We prove that

$$
d \Phi_{0}=3 \eta \wedge \Phi_{1}, \quad d \Phi_{1}=3 \eta \wedge \Phi_{2} .
$$


From (25), for all vector fields $X, Y, Z$ we have

$$
g\left(\left(\nabla_{X} \phi\right) Y, Z\right)=\eta(X) g(\phi h Y, Z)+\eta(Y) g(\phi h Z, X)+\eta(Z) g(\phi h X, Y),
$$

which implies that $d \Phi_{0}=3 \eta \wedge \Phi_{1}$. Analogously, from (26), we have

$$
g\left(\left(\nabla_{X} \phi h\right) Y, Z\right)=\eta(X) g\left(\phi h^{2} Y, Z\right)+\eta(Y) g\left(\phi h^{2} Z, X\right)+\eta(Z) g\left(\phi h^{2} X, Y\right),
$$

so that $d \Phi_{1}=3 \eta \wedge \Phi_{2}$. From (27),

$$
0=d^{2} \Phi_{0}=3 d \eta \wedge \Phi_{1}-3 \eta \wedge d \Phi_{1}=3 d \eta \wedge \Phi_{1} .
$$

Next we show that if 0 is a simple eigenvalue, then $\eta$ is a contact form. This, by an argument similar to the one in the proof of Theorem 3.3 will imply that $\operatorname{dim} M=5$.

First we assume that $\operatorname{Spec}\left(h^{2}\right)=\left\{0,-\lambda^{2}\right\}$, with $\lambda>0,0$ being a simple eigenvalue. This is equivalent to require that

$$
h^{2}=-\lambda^{2}(I-\eta \otimes \xi) .
$$

Let us take the tensor fields

$$
\tilde{\phi}=-\frac{1}{\lambda} h, \quad \tilde{\xi}=\frac{1}{\lambda} \xi, \quad \tilde{\eta}=\lambda \eta, \quad \tilde{g}=\lambda^{2} g .
$$

One can verify that $(\tilde{\phi}, \tilde{\xi}, \tilde{\eta}, \tilde{g})$ is an almost contact metric structure. Moreover, from 23 we have

$$
d \tilde{\eta}(X, Y)=2 \lambda g(h X, Y)=\frac{2}{\lambda} \tilde{g}(h X, Y)=2 \tilde{g}\left(X,-\frac{1}{\lambda} h Y\right)=2 \tilde{g}(X, \tilde{\phi} Y) .
$$

Therefore $(\tilde{\phi}, \tilde{\xi}, \tilde{\eta}, \tilde{g})$ is a contact metric structure. In particular, both the forms $\tilde{\eta}$ and $\eta$ are contact forms. Hence, in this case $M$ is a 5 -dimensional manifold and the multiplicity of the eigenvalue $-\lambda^{2}$ is 4 .

We assume now that

$$
\operatorname{Spec}\left(h^{2}\right)=\left\{0,-\lambda_{1}^{2}, \ldots,-\lambda_{r}^{2}\right\},
$$

where $\lambda_{i}$ is a positive real number and $\lambda_{i} \neq \lambda_{j}$ for $i \neq j$. From Proposition 4.3 . we know that for each $i=1, \ldots, r$, the distribution $[\xi] \oplus \mathcal{D}\left(-\lambda_{i}^{2}\right)$ is integrable with totally geodesic leaves. Each integral submanifold of this distribution is endowed with an induced almost contact metric structure, here again denoted by $(\phi, \xi, \eta, g)$, whose structure tensor field $h$ satisfies

$$
h^{2}=-\lambda_{i}^{2}(I-\eta \otimes \xi) .
$$

We deduce that $\eta$ is a contact form on the leaves of the distribution. In particular, each eigenvalue $-\lambda_{i}^{2}$ of $h^{2}$ has multiplicity 4 .

Notice that, taking two distinct eigenvalues $-\lambda_{i}^{2}$ and $-\lambda_{j}^{2}$, for every $X \in \mathcal{D}\left(-\lambda_{i}^{2}\right)$ and $Y \in \mathcal{D}\left(-\lambda_{j}^{2}\right)$, we have

$$
d \eta(X, Y)=2 g(h X, Y)=0,
$$

since the operator $h$ preserves the distributions $\mathcal{D}\left(-\lambda_{i}^{2}\right)$ and $\mathcal{D}\left(-\lambda_{j}^{2}\right)$, which are mutually orthogonal.

Now, fix a point $x \in M$. Since $\eta$ is a contact form on the leaves of each distribution $[\xi] \oplus \mathcal{D}\left(-\lambda_{i}^{2}\right)$, for any $i \in\{1, \ldots, r\}$ one can find a basis $\left(v_{1}^{i}, v_{2}^{i}, v_{3}^{i}, v_{4}^{i}\right)$ of $\mathcal{D}_{x}\left(-\lambda_{i}^{2}\right)$ such that

$$
\eta \wedge(d \eta)^{2}\left(\xi_{x}, v_{1}^{i}, v_{2}^{i}, v_{3}^{i}, v_{4}^{i}\right) \neq 0 .
$$

Therefore, putting $n=2 r$, the dimension of $M$ is $2 n+1$ and

$$
\begin{aligned}
\eta \wedge(d \eta)^{n} & \left(\xi_{x}, v_{1}^{1}, v_{2}^{1}, v_{3}^{1}, v_{4}^{1}, \ldots, v_{1}^{r}, v_{2}^{r}, v_{3}^{r}, v_{4}^{r}\right) \\
& =\eta\left(\xi_{x}\right)(d \eta)^{2}\left(v_{1}^{1}, v_{2}^{1}, v_{3}^{1}, v_{4}^{1}\right) \ldots(d \eta)^{2}\left(v_{1}^{r}, v_{2}^{r}, v_{3}^{r}, v_{4}^{r}\right) \neq 0 .
\end{aligned}
$$

This proves that $\eta$ is a contact form. 
Theorem 4.5. Let $(M, \phi, \xi, \eta, g)$ be a nearly cosymplectic non-coKähler manifold of dimension $2 n+1>5$. Then $M$ is locally isometric to one of the following Riemannian products:

$$
\mathbb{R} \times N^{2 n}, \quad M^{5} \times N^{2 n-4},
$$

where $N^{2 n}$ is a nearly Kähler non-Kähler manifold, $N^{2 n-4}$ is a nearly Kähler manifold, and $M^{5}$ is a nearly cosymplectic non-coKähler manifold.

Proof. If $h=0$, then $M$ is locally isometric to the Riemannian product $\mathbb{R} \times N^{2 n}$, where $N^{2 n}$ is a nearly Kähler non-Kähler manifold.

If $h \neq 0$, then $h^{2}$ admits non vanishing eigenvalues and we can assume $\operatorname{Spec}\left(h^{2}\right)=$ $\left\{0,-\lambda_{1}^{2}, \ldots,-\lambda_{r}^{2}\right\}$, where each $\lambda_{i}$ is a positive real number. Since $\operatorname{dim} M>5$, owing to Theorem 4.4, the eigenvalue 0 is not a simple eigenvalue. From b) and c) of Proposition 4.3, $M$ is locally isometric to the Riemannian product $M^{\prime} \times N$, where $M^{\prime}$ is an integral submanifold of the distribution $[\xi] \oplus \mathcal{D}\left(-\lambda_{1}^{2}\right) \oplus \ldots \oplus \mathcal{D}\left(-\lambda_{r}^{2}\right)$, and $N$ is an integral submanifold of $\mathcal{D}_{0}$, which is endowed with a nearly Kähler structure. Now, $M^{\prime}$ is endowed with an induced nearly cosymplectic structure for which 0 is a simple eigenvalue of the operator $h^{2}$. Therefore, by Theorem 4.4 we have that $\lambda_{1}=\ldots=\lambda_{r}$ and $M^{\prime}$ is a 5 -dimensional nearly cosymplectic non-coKähler manifold. Consequently, the dimension of $N$ is $2 n-4$.

Remark 4.6. Note that if the manifold $M$ in Theorem 4.5 is assumed to be complete and simply connected, then, by the de Rham decomposition theorem, the isometry becomes global as the involved distributions are parallel with respect to the Levi-Civita connection. Note also that the nearly Kähler factor can be further decomposed. See Theorem 1.1 and Proposition 2.1 in [14] for details.

\section{REFERENCES}

[1] D. E. Blair, Almost contact manifolds with Killing structure tensors, Pacific J. Math. 39 (1971), no. 2, 285-292.

[2] D. E. Blair, Riemannian geometry of contact and symplectic manifolds. Second Edition. Progress in Mathematics 203, Birkhäuser, Boston, 2010.

[3] D. E. Blair, D. K. Showers, Almost contact manifolds with Killing structure tensors. II., J. Differential Geom. 9 (1974), 577-582.

[4] D. E. Blair, D. K. Showers, K. Yano, Nearly Sasakian structures, Kodai Math. Sem. Rep. 27 (1976), no. 1-2, 175-180.

[5] C. P. Boyer, K. Galicki, Sasakian geometry, Oxford University Press, 2008.

[6] B. Cappelletti-Montano, A. De Nicola, I. Yudin, A survey on cosymplectic geometry, Rev. Math. Phys. 25 (2013), no. 10, 1343002, 55 pages.

[7] B. Cappelletti-Montano, G. Dileo, Nearly Sasakian geometry and SU(2)-structures, Ann. Mat. Pura Appl. (IV) 195 (2016), 897-922.

[8] D. Chinea, C. Gonzalez, A classification of almost contact metric manifolds, Ann. Mat. Pura Appl. (IV) 156 (1990), 15-36.

[9] H. Endo, On the curvature tensor of nearly cosymplectic manifolds of constant $\phi$-sectional curvature, An. Stiit. Univ. "Al. I. Cuza "Iasi. Mat. (N.S.) 51 (2005), 439-454.

[10] H. Endo, On the first Betti number of certain compact nearly cosymplectic manifolds, J. Geom. 103 (2012), no. 2, 231-236.

[11] J. C. González-Dávila, F. Martín Cabrera, Harmonic almost contact structures via the intrinsic torsion, Israel J. Math. 181 (2011), 145-187.

[12] A. Gray, The structure of nearly Kähler manifolds, Math. Ann. 223 (1976), 233-248.

[13] E. Loubeau, E. Vergara-Diaz, The harmonicity of nearly cosymplectic structures, Trans. Amer. Math. Soc. 367 (2015), 5301-5327.

[14] P. A. Nagy, Nearly-Kähler geometry and Riemannian foliations, Asian J. Math. 6 (2002), no. 3, 481-504.

[15] Z. Olszak, Nearly Sasakian manifolds, Tensor (N.S.) 33 (1979), no. 3, 277-286.

[16] Z. Olszak, Five-dimensional nearly Sasakian manifolds, Tensor (N.S.) 34 (1980), no. 3, 273276.

[17] E. Vergara-Diaz, C. M. Wood, Harmonic almost contact structures, Geom. Dedicata 123 (2006), 131-151. 
Dipartimento di Matematica, Università degli Studi di Salerno, Via Giovanni Paolo II 132, 84084 Fisciano, ITALY

E-mail address: antondenicola@gmail.com

Dipartimento di Matematica, Università degli Studi di Bari Aldo Moro, Via E. Orabona 4, 70125 BARI, ITALY

E-mail address: giulia.dileo@uniba.it

CMUC, Department of Mathematics, University of Coimbra, 3001-501 Coimbra, PorTUGAL

E-mail address: yudin@mat.uc.pt 